ARTICLE

\title{
Reaction inhomogeneity coupling with metal rearrangement triggers electrochemical degradation in lithium-rich layered cathode
}

\author{
Liguang Wang (10 1,2, Tongchao Liu ${ }^{3}$, Alvin Dai ${ }^{3}$, Vincent De Andrade ${ }^{4}$, Yang Ren (1D ${ }^{4}$, Wenqian Xu ${ }^{4}$, \\ Sungsik Lee ${ }^{4}$, Qinghua Zhang ${ }^{5}$, Lin $\mathrm{Gu}^{5}$, Shun Wang (1D ${ }^{1}$, Tianpin Wu ${ }^{4 凶}$, Huile Jin ${ }^{1 凶} \&$ Jun Lu (i) ${ }^{3 凶}$
}

High-energy density lithium-rich layered oxides are among the most promising candidates for next-generation energy storage. Unfortunately, these materials suffer from severe electrochemical degradation that includes capacity loss and voltage decay during long-term cycling. Present research efforts are primarily focused on understanding voltage decay phenomena while origins for capacity degradation have been largely ignored. Here, we thoroughly investigate causes for electrochemical performance decline with an emphasis on capacity loss in the lithium-rich layered oxides, as well as reaction pathways and kinetics. Advanced synchrotron-based $\mathrm{X}$-ray two-dimensional and three-dimensional imaging techniques are combined with spectroscopic and scattering techniques to spatially visualize the reactivity at multiple length-scales on lithium- and manganese-rich layered oxides. These methods provide direct evidence for inhomogeneous manganese reactivity and ionic nickel rearrangement. Coupling deactivated manganese with nickel migration provides sluggish reaction kinetics and induces serious structural instability in the material. Our findings provide new insights and further understanding of electrochemical degradation, which serve to facilitate cathode material design improvements.

\footnotetext{
${ }^{1}$ Key Laboratory of Carbon Materials of Zhejiang Province, Institute of New Materials and Industrial Technologies, Wenzhou University, Wenzhou, Zhejiang China. ${ }^{2}$ Department of Chemistry and Biochemistry, University of Windsor, Windsor, ON, Canada. ${ }^{3}$ Chemical Sciences and Engineering Division, Argonne National Laboratory, Lemont, IL, USA. ${ }^{4}$ X-ray Science Division, Advanced Photon Sources, Argonne National Laboratory, Lemont, IL, USA. ${ }^{5}$ Beijing National

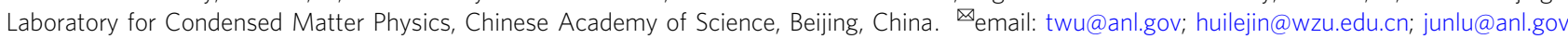


O ne of the primary challenges for modern lithium-ion batteries (LIBs) development is realizing high-energydensity cathode materials that satisfy increasing demands for long range electric vehicles. Lithium- and manganese-rich (LMR) oxides are promising candidates due to their noteworthy energy density that exceeds $900 \mathrm{Wh} \mathrm{Kg}^{-1}$ (vs. Li metal) $)^{1,2}$. Nevertheless, these materials suffer from serious electrochemical degradation, including voltage decay and capacity loss, which restricts real-world implementation ${ }^{3,4}$. Efforts to elucidate the origins of voltage decay have attributed unstable, irreversible, phase transitions from layered structures to spinel-like or rocksalt phases, induced by transition metal (TM) migration from TM layers to lithium layers, as the root cause $e^{5,6}$. Despite rising concern for capacity degradation and its close correlation to electrode reaction kinetics, its mechanisms and limitations placed on largescale implementation remain unaddressed.

Efficient reaction kinetics are critical for electrode design and have profound effects on overall electrochemical performance ${ }^{7,8}$. Electrode reaction dynamics can also be influenced by many factors at multiple length scales. For example, increased cationic intermixing can block lithium diffusion pathways in the lattice structure $^{1,9}$, and irreversible phase transformations caused by oxygen release reduce electrochemically active surfaces within cathode particles ${ }^{10}$. In turn, these atomic structural and chemical defects can aggravate charge heterogeneity inside the particle, inducing severe strains and stresses that exacerbate mechanical electrode deterioration ${ }^{11}$. Although tremendous efforts have been taken to suppress thermodynamic instability in LMR materials through methods such as cation doping ${ }^{12,13}$ and surface modification $^{14,15}$, correlations between capacity degradation and the evolution of reaction kinetics remain barely studied. Given that LMR electrodes exhibit intrinsically sluggish anionic redox when compared with common ternary materials ${ }^{16}$, it is all the more necessary to reveal electrochemical degradation mechanisms with respect to reaction dynamics, especially at multiple length scales from atomic to particle and bulk/electrode levels.

In this work, we directly capture spatial structure evolutions in high-capacity $\mathrm{Li}_{1.2} \mathrm{Ni}_{0.13} \mathrm{Co}_{0.13} \mathrm{Mn}_{0.54} \mathrm{O}_{2}$ (LR-NCM) cathodes during long-term cycling using advanced synchrotron-based $\mathrm{X}$ ray imaging techniques and $\mathrm{X}$-ray absorption near edge structures (XANES). These techniques highlight the different behaviors of various transition metals (nickel and manganese) in the secondary particle level, revealing deactivated and correlated anisotropic reactivity for manganese and ionic rearrangement for nickel. Induced by asynchronous solid reactions, large strain and grain boundaries formed between primary crystals produce crack formations in LR-NCM particles. Subsequent structural changes at atomic levels and bulk averages were systematically studied to elucidate their contributions to electrochemical degradation, which include voltage decay, voltage hysteresis, and specially, capacity decrease. Electrochemical instability was found to be closely related to manganese deactivation and anisotropic reactions. When combined with nickel rearrangement, this instability results in electrochemical degradation of LR-NCM cathodes. These new insights into the distinct behaviors of LR-NCM transition metals elucidate the origins of capacity degradation, voltage decay, and facilitate the development of high-capacity cathode designs.

\section{Results}

Electrochemical properties degradation. The electrochemical properties of LR-NCM material are shown in Fig. 1 and Supplementary Fig. 1. Initial charge profile (Supplementary Fig. 1a) shows a voltage plateau around $4.5 \mathrm{~V}$ that indicates anionic redox features in the LR-NCM material ${ }^{17}$. The material also exhibits
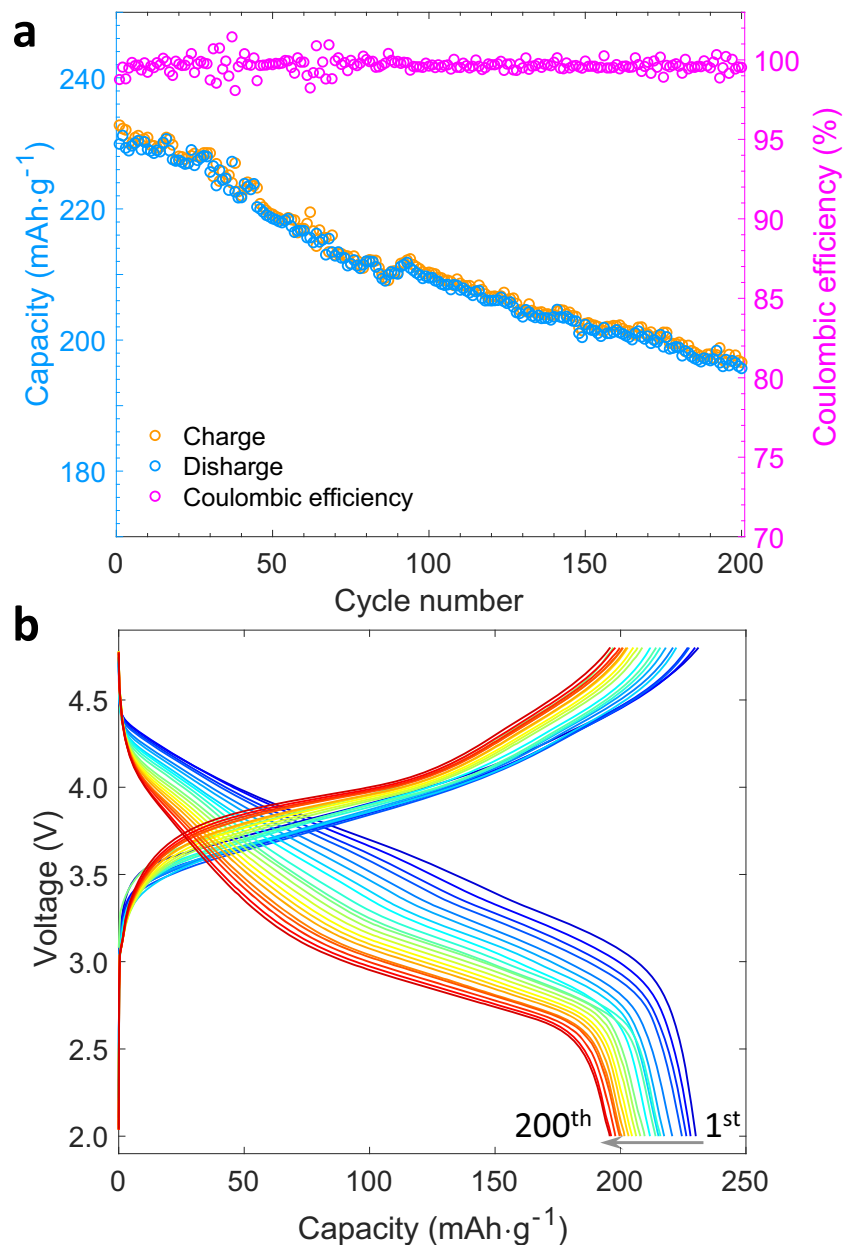

Fig. 1 Cycling stability of LR-NCM. a Cycling performance of LR-NCM electrode at a current of $0.3 \mathrm{C}$. b Corresponding charge-discharge profiles from $1^{\text {st }}$ to $200^{\text {th }}$ cycle.

dramatic capacity degradation with a discharge capacity retention of $85 \%$ as shown in Fig. 1 after 200 charge-discharge cycles at a current rate of $0.3 \mathrm{C}$ (Fig. 1). As mentioned above, this capacity decrease combined with serious voltage decay limits further commercial applications. Currently, researchers primarily focus on voltage decay phenomena (illustrated in Supplementary Fig. $1 \mathrm{~b}-\mathrm{d}$ ), which is correlated to thermodynamic changes caused by phase transformation ${ }^{18}$. However, key mechanisms for capacity degradation induced by structure effects and evolving reaction kinetics have been seldomly studied systematically. Herein, we employ multiple advanced characterization techniques to provide insights into the electrochemical degradation of LRNCM.

Revealing the reaction homogeneity in $\mathrm{Mn}$ and $\mathrm{Ni}$. To understand the reaction homogeneity in TMs spatially, we first employed advanced synchrotron-based full-field transmission X-ray microscopy (TXM) technique combined with XANES spectroscopy to directly observe the distribution of $\mathrm{Ni}$ and $\mathrm{Mn}$ related chemical phases on the particle level (Fig. 2). The twodimensional (2D) TXM-XANES mappings give the aging states mapping for each element analyzed based on the changes in XANES spectra (see detailed data processing in "Methods" and Supplementary Fig. 2). This changing in XANES spectra corresponds to the aging state of TM-based phase, which contains the 


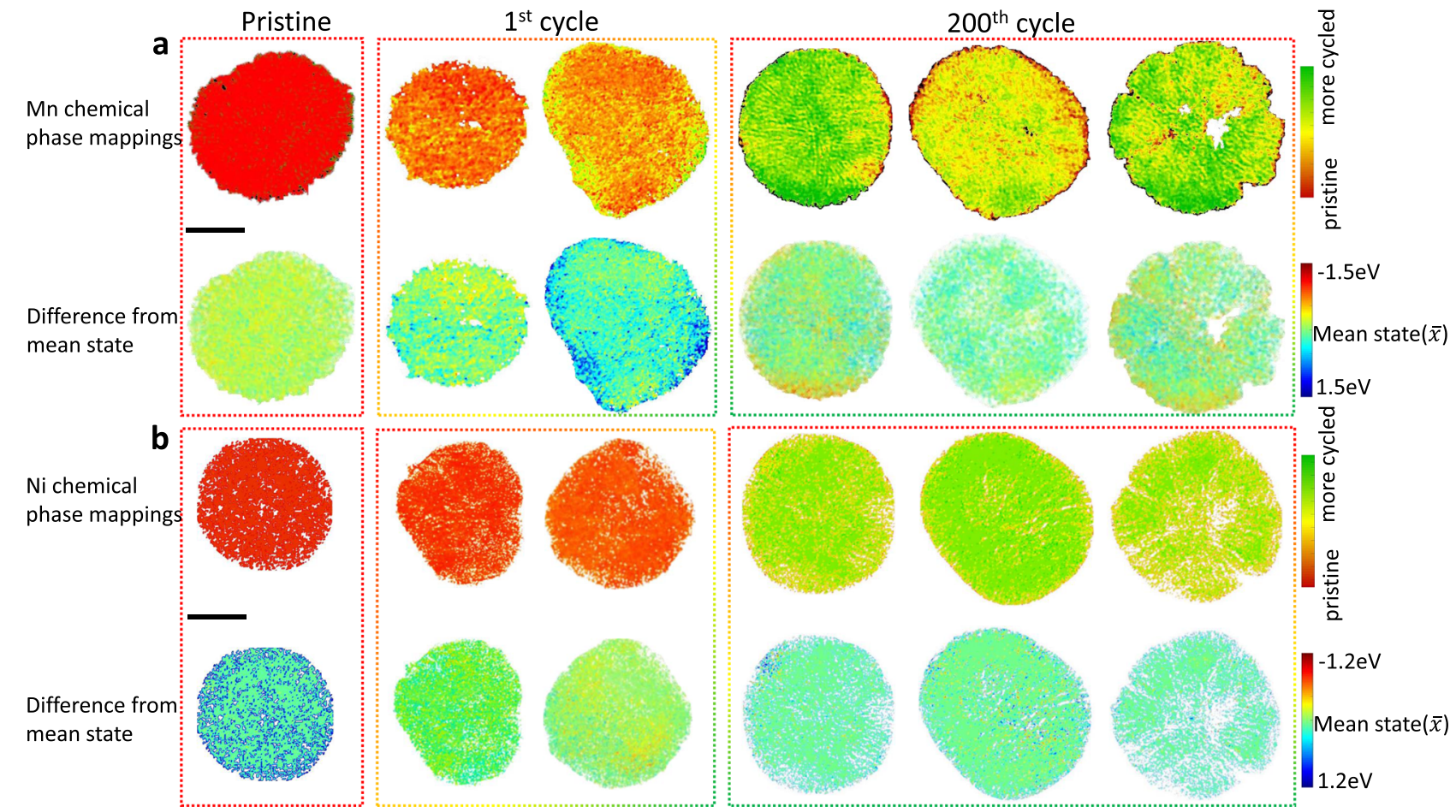

Fig. 2 Anisotropic reaction characterized by TXM-XANES technique. 2D chemical phase mappings of LRst th NCM particles at a Mn K-edge and $\mathbf{b}$ Ni K-edge, respectively. Three states including pristine, $1^{\text {st }} \mathrm{cycle}$, and $200^{\text {th }} \mathrm{cycle}$, were selected. The top figure corresponds to the chemical phase mappings and the bottom row figure represents the difference from the corresponding average state. Scale bar: $5 \mu \mathrm{m}$.

electronic structural and local structural (or ligands affects around the metals) evolutions. For the Mn-related chemical phase (Fig. 2a and Supplementary Fig. 3a), chemical phase mappings (top figures) and phase difference mappings from bulk averages (bottom figures) demonstrate uniform phase distribution in the pristine state. However, phase propagation is rather anisotropic after long-term cycling and identified with distinct color coding. Prior research has proven that $\mathrm{Mn}$ in the pristine state is electrochemically inactive, and gradually becomes active via $\mathrm{Mn}^{3+/ 4+}$ redox during the first few cycles ${ }^{19,20}$. Activated $\mathrm{Mn}^{3+/ 4+}$ redox proceeds through anionic chemistry and provides excess capacity in subsequent cycles. Large red regions in particles after 200 cycles indicate reversal back to the inactive state, which we believe is one of the major reasons for electrochemical degradation. This increase in deactivated $\mathrm{Mn}$ ions could further aggravate charge heterogeneity and result in sluggish reaction kinetics or even isolation from the conducting network. Red regions (deactivated phase) are mostly distributed on particle surfaces and interior particles near pores, while cracks form during cycling. This phenomenon is highly correlated to chemical instability between the LR-NCM and the electrolyte as proposed by many previous works 7,21 .

As opposed to the anisotropic reactivity of $\mathrm{Mn}, \mathrm{Ni}$ reactions exhibit high reversibility during long-term cycling (Fig. $2 \mathrm{~b}$ and Supplementary Fig. 3b). Consistent color regions distributed evenly on Ni surfaces and crack formation indicate that there is no obvious chemical phase separation after 200 cycles, unlike in the $2 \mathrm{D}$ chemical phase mappings of $\mathrm{Mn}$. This means $\mathrm{Ni}$ ion aging states are uniform in whole secondary particles, indicating the isotropic reactivity of $\mathrm{Ni}$ in LR-NCM during cycling. However, local structures around $\mathrm{Ni}$ ions are altered between pristine and cycled states, which could affect reactivity (discussed later in detail). The different reaction behavior between $\mathrm{Mn}$ and $\mathrm{Ni}$ recognized by TXM-XANES demonstrates that inhomogeneous reactions in Mn-related phases enhance material deterioration. Owing to the asynchronous reactivity in TMs, aggravating deactivated Mn-domains could also induce significant strain within and between crystals, leading to the formation of grain boundaries and cracks. Thus, it is essential to further investigate the chemo-mechanical changes in LR-NCM during cycling.

Chemo-mechanical changes investigated by energy-resolved nano-tomography. Three-dimensional (3D) chemo-mechanical changes were characterized by synchrotron-based energyresolved X-ray nano-tomography (see details in the experimental section $)^{22,23}$. Uniform $\mathrm{Ni}$ distribution in the whole secondary particle is confirmed by homogenous color distributions in Supplementary Fig. 4. The prepared LR-NCM exhibits spherical morphology with porous structures that are intentionally introduced to buffer volume changes during battery operation. Unfortunately, cracks still formed (Fig. 3a-e and Supplementary Movie 1) due to repeated volume changes that were coupled with large strains caused by anisotropic reactions as mentioned above. For particles after 200 cycles, the color-coded figure shows distinct color regions when compared to the pristine material, which indicates $\mathrm{Ni}$ segregation, rearrangement, and aggregation at red regions (Fig. 3b and Supplementary Fig. 5). These changes could induce serious irreversible phase transformation and lead to the observed voltage decay. Moreover, Ni dissolution phenomenon involving $\mathrm{Ni}^{2+}$ leaching into the electrolyte is also directly observed as a blue circle that appears around the spherical particle (Fig. $3 \mathrm{c}$ and Supplementary Fig. 5). This blue circle corresponds to low $\mathrm{Ni}$ concentrations observed beyond the active material, which can explain the decreased reduction peak intensity of $\mathrm{Ni}^{4+/ 2+}$ (losing capacity) in the differential capacity curves (Supplementary Fig. 1c).

In contrast, neither metal segmentation nor obvious dissolution was observed in Mn after long-term cycling (Fig. 3d, e and 


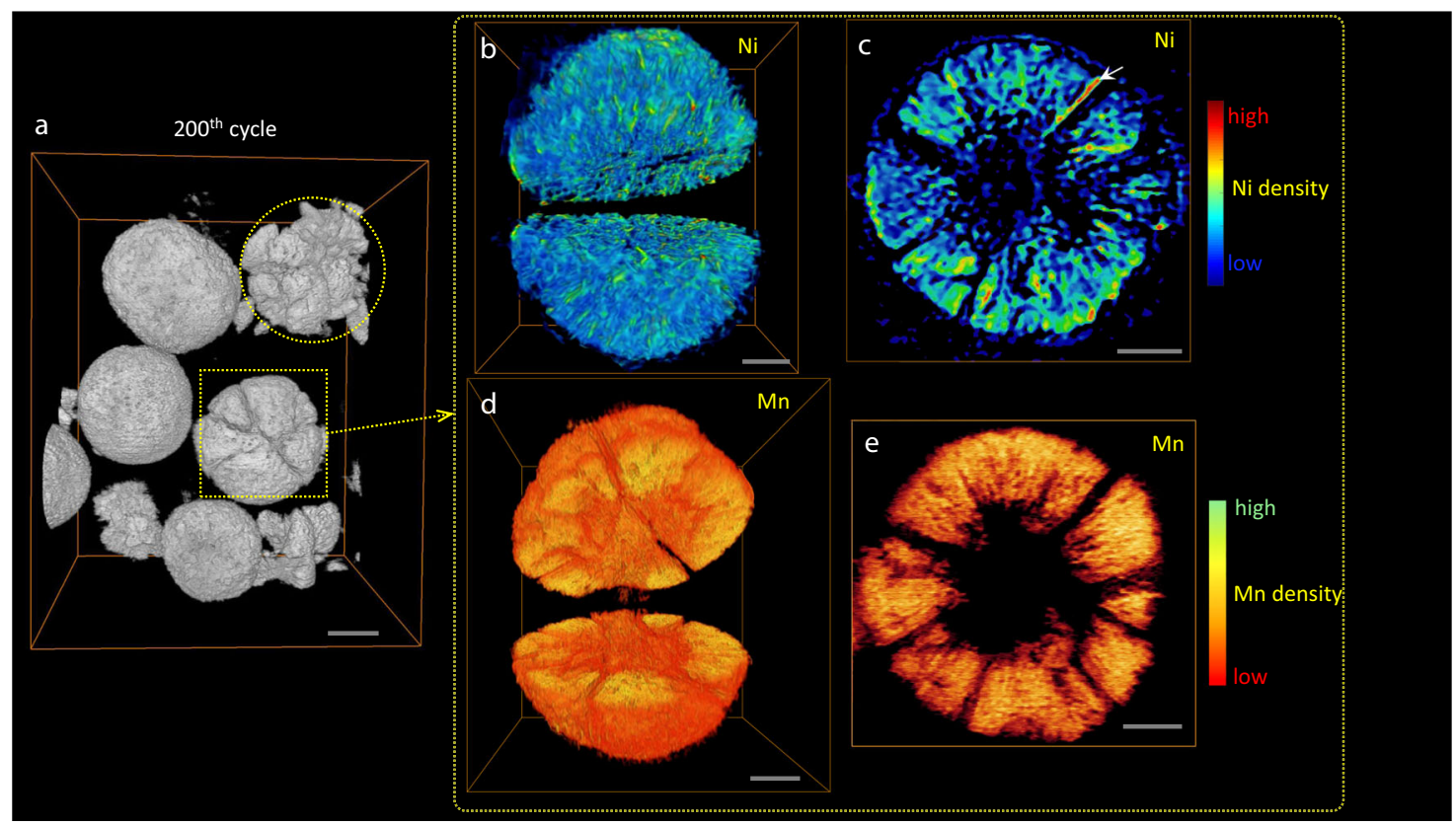

Fig. 3 Elemental distribution visualized via 3D nano-tomography. a The large view of 3D nano-tomography after cycling. Scale bar: $5 \mu \mathrm{m}$. b-e 3D open inside view and the corresponding cross-sectional view of the selected particle in $\mathbf{a}$. The colorbar on the right shows the concentration of the corresponding element (Ni: b-c Mn: d-e). Scale bar: $2 \mu \mathrm{m}$.

Supplementary Fig. 6). We selected the same particle as characterized in Ni for in-depth analysis (Fig. 3d), while another one with comparable morphology is shown in Supplementary Fig. 6. Color-coded mapping demonstrates homogenous color regions with an average concentration of $\mathrm{Mn}$ that indicates uniform elemental distribution inside the particles. Unlike some nickel emerging surrounding the particle as a blue circle in Fig. $3 c$, there is no clear manganese signals showing outside the selected particle. Consequently, we did not observe the obvious $\mathrm{Mn}$ dissolution phenomenon that usually shows in other Mnbased materials ${ }^{24}$. This suggests $\mathrm{Mn}$ works as a stabilizer in the solid. Compared to $\mathrm{Ni}, \mathrm{Mn}$ in $\mathrm{Li}_{2} \mathrm{MnO}_{3}$-like domains rearranging with LiMn2 ordering remain highly stable due to average oxidation states of over $3+$ even after the activation process in the first few cycles. Theoretically, $\mathrm{Mn}$ does not experience chemical instability because the $\mathrm{Mn}^{3+/ 4+}$ band is higher and does not overlap the $\mathrm{O}^{2-} 2 p$ band ${ }^{3}$. This finding for high stability of $\mathrm{Mn}$ in the lattice can provide fundamental evidence for the capability of LR-NCM to stabilize at voltages as high as $4.8 \mathrm{~V}$, which is much greater than that of traditional ternary cathode materials (NCMs).

Atomic characterization of structural changes. High-resolution scanning transmission electron microscopy (STEM) was performed on the particles at pristine states and after degradation to further investigate structural changes at the atomic level. Figure $4 \mathrm{a}$ demonstrates the perfect atomic arrangement as a layered structure for LR-NCM in the [110] plane. However, after longterm cycling, there is a consensus that serious phase transitions to spinel/rock-salt structures occur on secondary particle surfaces (Supplementary Fig. 7). Our TXM-XANES results show chemical phase deactivation even inside the secondary particle. To confirm this degradation, we further investigated the atomic structure in core particle areas (Fig. 4b). Multiple phases at different states and phase transformations from layered structure to spinel-like/ rock-salt phase were observed (Fig. 4c-e). A large fully transformed rock-salt phase is demonstrated along the [111] plane with FFT imaging (Fig. 4c). A phase with severe Li/TM intermixing is also observed on other crystals in the core area (Fig. 4d). These atomic structural evolutions are closelycorrelated to the stability of oxygen. When oxygen redox, O-O dimer formation along with oxygen movement can dramatically change local environment around $\mathrm{TMs}^{25}$, which could affect the metal rearrangement mentioned above. Agree well with previous work $^{26}$, the irreversible phase transformation can be described in multiple gradual steps (Fig. 4e): (i) TMs migrate to vacant Li sites; (ii) the new slab shifts $1 / 3$ along the [110] plane; (iii) spinel-like phases are formed with oxygen release. In addition, a disordered phase that is nearly amorphous appears on the particle surface, which is correlated to $\mathrm{Ni}$ dissolution from the solid and the formation of cathode electrolyte interfaces. Apart from these irreversible phase transformations, it cannot be neglected for the local structural evolutions in terms of high-capacity correlated superstructures in LR-NCM. Pristine material shows clear LiMn2 ordering as investigated by STEM (Supplementary Fig. 8) and the two-phase model XRD refinement (Supplementary Fig. 9 and Supplementary Table 1). Such ordering structure exists in the crystal activates the oxygen redox at high voltages, which is the primary reason for the high capacity. Therefore, the disappearing ordering structure over cycling is also considered as one of the most important factors leading to the capacity degradation ${ }^{27}$. This local structural ordering changes interacted with the abovementioned phase transformations aggravate the structural irreversibility over cycling. These irreversible phase transformations accumulate into large inactive domains that hinder lithium diffusion and electron transfer, which causes degradation and electrode fatigue.

Electronic and local structure characterized by X-ray absorption spectroscopy. X-ray absorption spectroscopy at $\mathrm{Ni}$ and $\mathrm{Mn}$ $K$-edge was conducted to reveal the origin of anisotropic reactions. The XANES spectra of Ni K-edge remain close to that of 

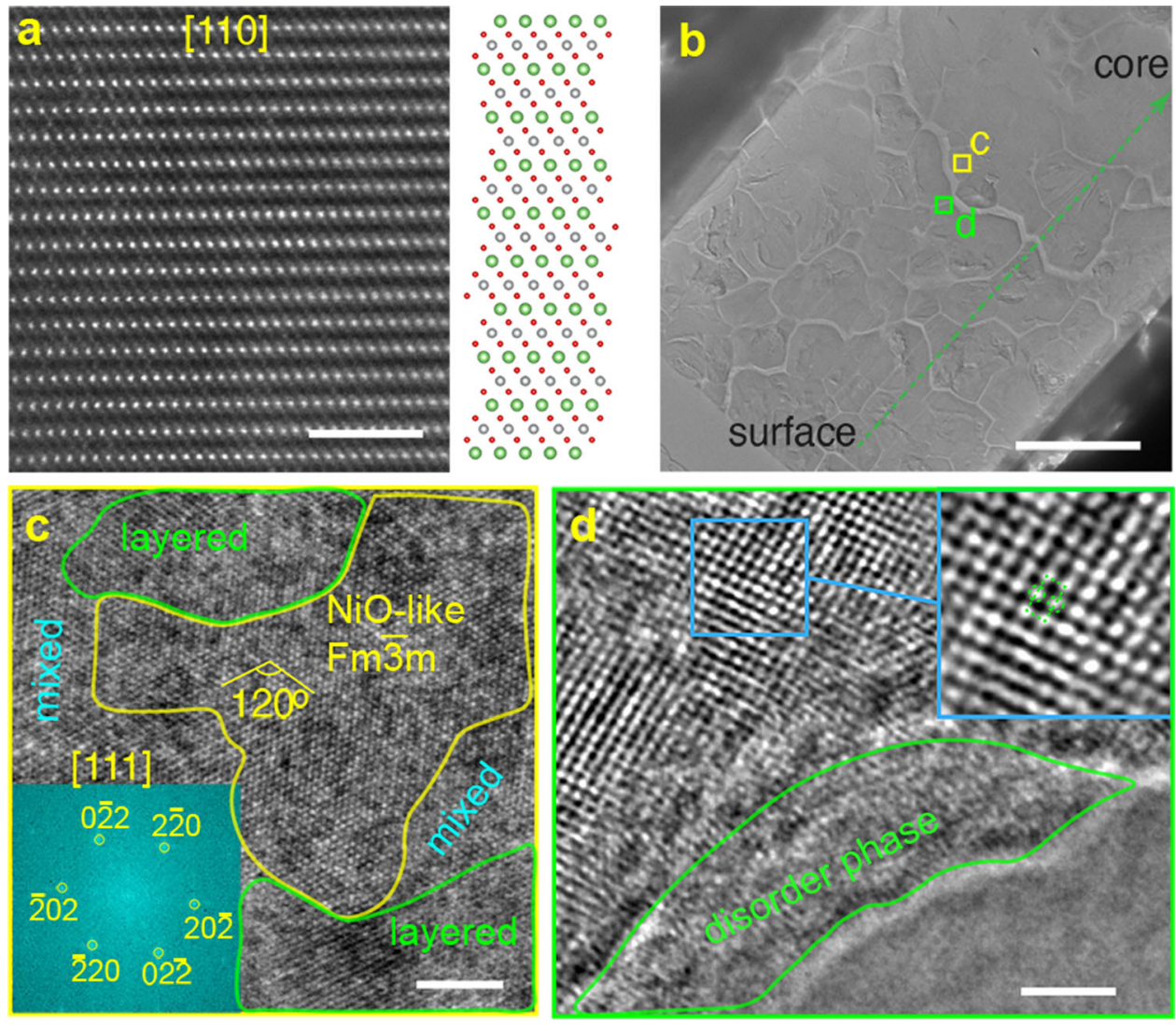

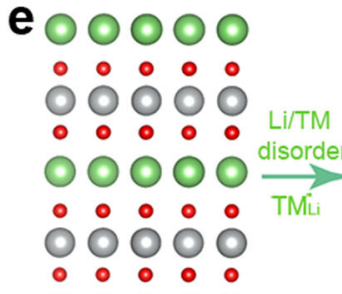

00000

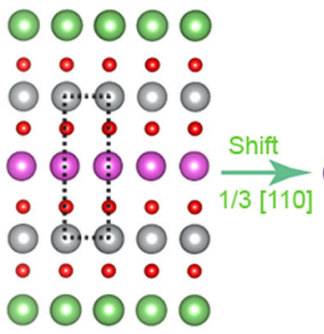

O Li

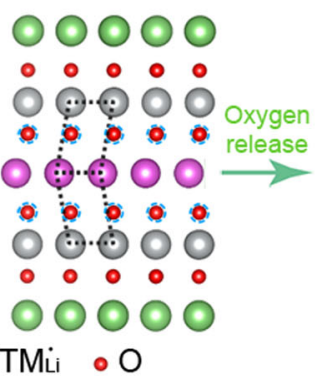

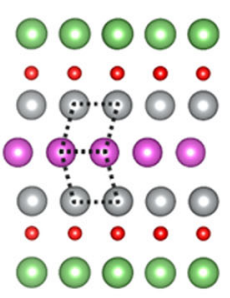

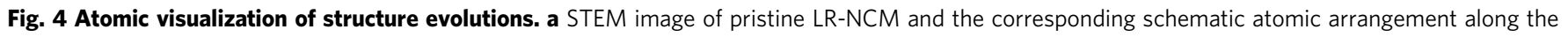

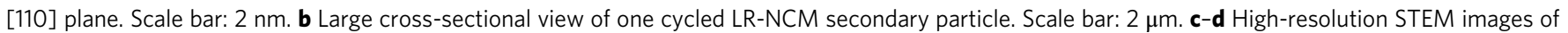

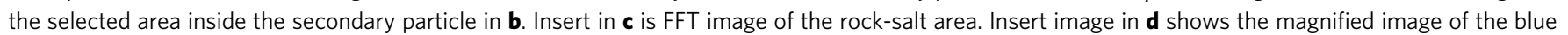
region. Scale bar: $2 \mathrm{~nm}$ e Schematic illustration of structural evolutions from layered structure to rocksalt phase.

standard $\mathrm{NiO}$ (Fig. 5a) except for shifts observed in the vicinity of $\mathrm{Ni}$, as indicated by the white line (Fig. $5 \mathrm{c}$ ). This indicates the valence of $\mathrm{Ni}$ in LR-NCM is still $2+$ when cycled and further electrochemical reactions are possible. Compared to elemental $\mathrm{Ni}$, Mn $K$-edge XANES spectra changes much more owing to its anisotropic reactivity over cycling as discussed above (Supplementary Fig. 10a). The absorption of pristine LR-NCM at Mn Kedge along the white line is similar to that of $\mathrm{Li}_{2} \mathrm{MnO}_{3}$, indicating valences close to $4+$ which is in agreement with previously reported results ${ }^{28}$. However, determining the valence state of $\mathrm{Mn}$ ions accurately only by comparison of the profiles along the white line is difficult, owing to the complex Mn K-edge spectra. Part of the Mn XANES spectra of the aged electrode is close to that of $\mathrm{MnO}_{2}$, which demonstrates significant differences in local environments.

In order to probe local structure evolution around $\mathrm{Ni}$ and $\mathrm{Mn}$, we further conducted quantitative analysis of the extended X-ray absorption fine structure (EXAFS) results (Supplementary
Fig. 10b-d). The average bond lengths in the first two shells display no obvious variation when cycled (Supplementary Fig. 10c), except for slightly increased $M-M$ bond lengths in the second shell. This indicates a consistent host solid structure, while small lattice extensions along the $a(b)$ direction are explainable by increased lattice parameters determined with XRD refinement (Supplementary Fig. 9b, c). In pristine LR-NCM, the first shell of oxygen demonstrates full occupation for TMs and a coordination number $(\mathrm{CN})$ for $\mathrm{M}-\mathrm{O}$ of six, whereas the content in the second shell shows large differences between $\mathrm{Ni}$ and $\mathrm{Mn}$. The second shell shows a decreased $\mathrm{CN}$ of $\mathrm{Mn}-\mathrm{M}$ from six to three owing to the substitution of $\mathrm{Mn}$ by $\mathrm{Li}$ in the $\mathrm{Li}_{2} \mathrm{MnO}_{3}$-like domains. There is a consensus that oxygen release leaves many vacancies during cycling, which induces the migration of $\mathrm{Ni}$ ions from the center of the octahedral to the tetrahedron site. The decreased intensity of the higher order shells at distances over $3 \AA$ represents the changed $\mathrm{Ni}$ ionic arrangement. In contrast, structures around $\mathrm{Mn}$ ions in this scattering range display more 

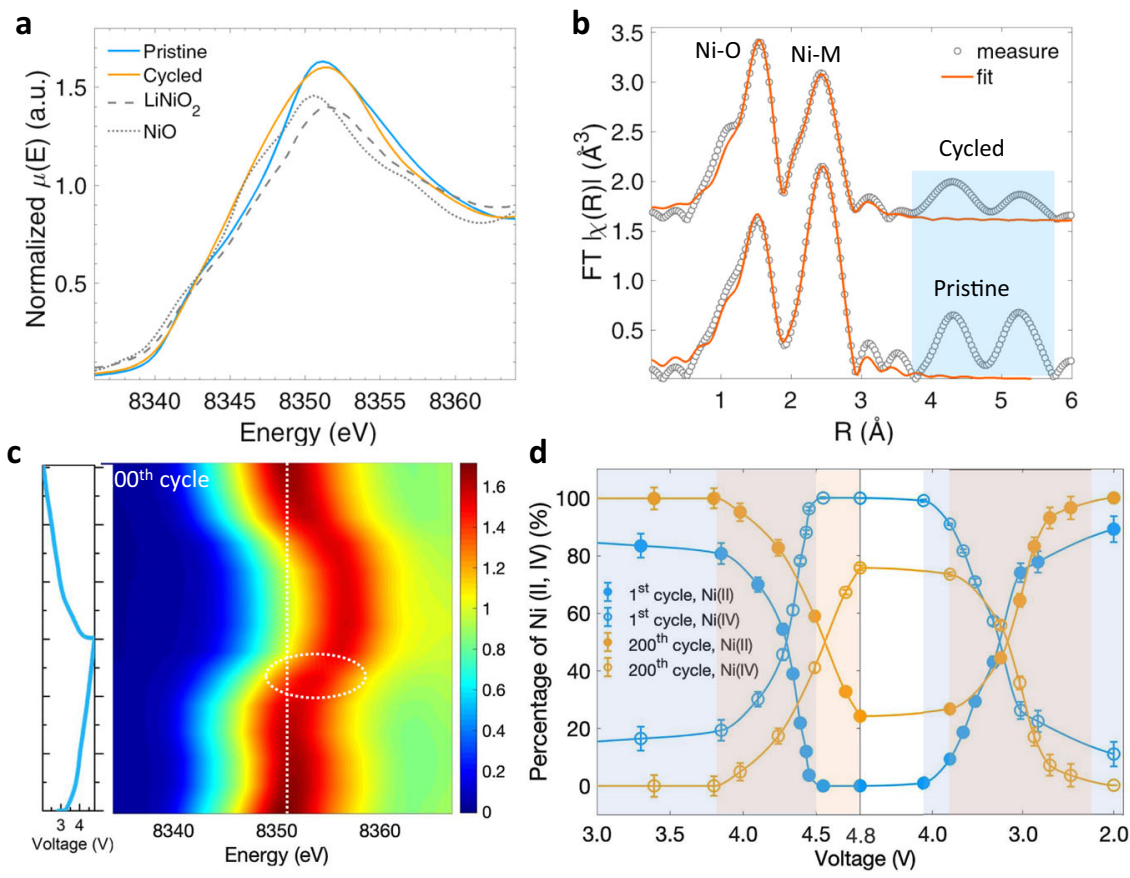

Fig. 5 The changing reactivity of Ni. a Comparison of the XANES spectra and $\mathbf{b}$ the fitting results of Fourier transformed EXAFS curves of pristine and cycled electrodes at $\mathrm{Ni}$ K-edge. c Contour plot of in-operando XANES spectra at Ni K-edge during the 200th charge-discharge processes. $\mathbf{d}$ Quantified analysis of $\mathrm{Ni}^{2+4+}$ redox at the first and $200^{\text {th }}$ cycle. Error is obtained by the linear combination fitting of each spectrum.

stable behavior as evidenced in the similar FT $\chi \mathrm{R})$. The contrasting evolutions of electronic and local structure between $\mathrm{Ni}$ and $\mathrm{Mn}$ determine the degradation mechanism of $\mathrm{Ni}$ rearrangement and $\mathrm{Mn}$ deactivation, which is in good agreement with TXM-XANES and 3D nano-tomography results.

The evolution of reaction mechanisms. We now demonstrated how electrochemical processes in LR-NCM after long-term cycling are affected by increasing anisotropic reactions and metal rearrangement. In order to further probe these effects, we investigated the electronic chemistry in $\mathrm{Ni}$ and $\mathrm{Mn}$ elements during the $200^{\text {th }}$ charge-discharge process by using in-operando XANES technique (Fig. 5c, d and Supplementary Fig. 11). Although contour plots demonstrate the full reversibility of $\mathrm{Ni}$, quantitative analysis of the oxidation state as a function of voltage shows significant differences between the first and $200^{\text {th }}$ cycle (Fig. 5d). $\mathrm{Ni}^{2+}$ is fully oxidized to the highest oxidation state of $4+$ at $4.5 \mathrm{~V}$ during the first charge, exhibiting no electrochemical activation at further high voltage. Meanwhile, the oxidation of $\mathrm{Ni}^{2+}$ occurs at higher average voltages in the aged electrode, which means the reaction barrier energy increased dramatically, leading to a large electrochemical reaction overpotential $(\eta)$. In addition, average $\mathrm{Ni}$ oxidation states are lower than $4+$ at the fully charged state and can be attributed to two factors that may also explain capacity degradation. One reason is that large $\eta$ leads to serious voltage hysteresis and sluggish reaction kinetics that result in only partial $\mathrm{Ni}^{2+}$ oxidation at the same cutoff voltage of $4.8 \mathrm{~V}$. Another is that a fraction of $\mathrm{Ni}^{2+}$ is involved in the formation of electrochemical inactive NiO-like phases. The discharge process demonstrates contrasting behavior, where the reduction of $\mathrm{Ni}^{4+}$ in the aged electrode takes place at lower voltages compared to the first cycle. Interestingly, although $\mathrm{Mn}$ is partially deactivated after cycling, the overall reaction showed active $\mathrm{Mn}^{3+/ 4+}$ redox with high reversibility (Supplementary Fig. 11). The growth in the intensity of the second pre-edge peak corresponds to the emptying of electrons in the $e_{g}$ level, which suggests the oxidation of $\mathrm{Mn}^{3+}$ to $\mathrm{Mn}^{4+29}$. Unlike during the first cycle, the pre-edge peaks of in-operando Mn K-edge XANES spectra during the whole $200^{\text {th }}$ cycle change around one peak state as shown in Supplementary Fig. 10, indicating a small range valence evolution. This phenomenon confirms the presence of electrochemically deactivated $\mathrm{Mn}$ after degradation, which explains LR-NCM capacity loss.

In-operando long-duration synchrotron $\mathrm{XRD}$ was carried out on the LR-NCM cathode at the first and $201^{\text {st }}$ cycles to understand the evolution of the aged LR-NCM (Fig. 6a and Supplementary Figs. 13, 14). During initial charging (003) reflection shifts gradually to lower angles, which corresponds to increase in $c$-spacing (Fig. 6b) induced by the growing repulsive force between two oxygen layers after lithium extraction. However, this process is put off to even higher voltages in the aged electrode (Fig. 6b) due to the large energy barrier caused by increased cationic intermixing as proved above. Slightly splitting of (015) reflection (Supplementary Fig. 14b) also proved the restricted $\mathrm{H} 1-\mathrm{H} 2$ transformation, indicating the losing reactivity in TMs. Given the small changes in the diffraction patterns, further follow-up studies to clarify this peak splitting is necessary to clearly demonstrate the phase transition. C-axis values correspond to state-of-charge (SoC) changes, and smaller variations at low voltages during the $201^{\text {st }}$ cycle explain the origin of capacity loss as the result of metal migration. The narrow structural evolution at high-voltage range (yellow area in Fig. 6b) caused by the large overpotential in the aged electrode demonstrates another part of capacity degradation. In addition, the unit cell volume $(V)$ exhibits nearly two times increase in magnitude for the aged electrode $(2.0 \%)$ than in the first cycle $(1.1 \%)$. This large volume evolution when cycled could further aggravate stress inside the solid, resulting in growing grain boundaries and crack formations as observed by 3D nanotomography. Note that we only applied one-phase model in inoperando XRD patterns refinement to avoid the deviations arising 
a

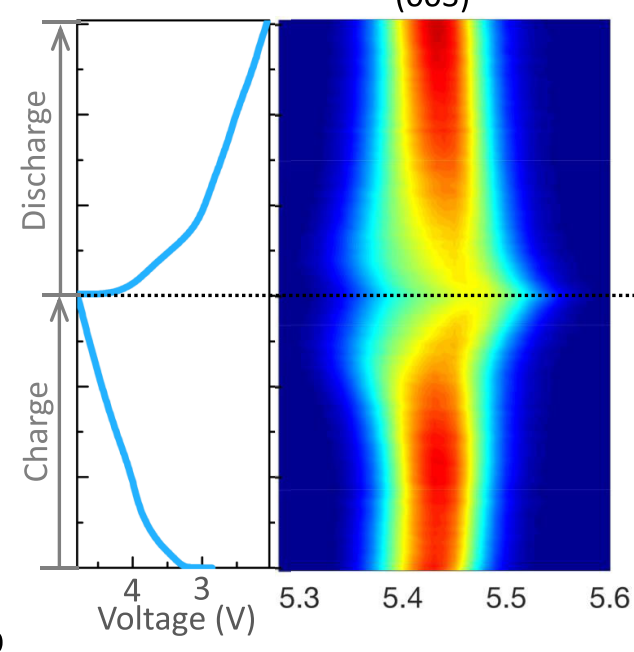

$(01 \overline{1})$

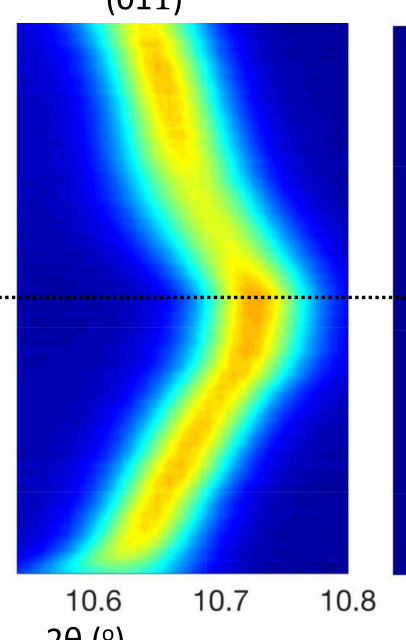

(018)

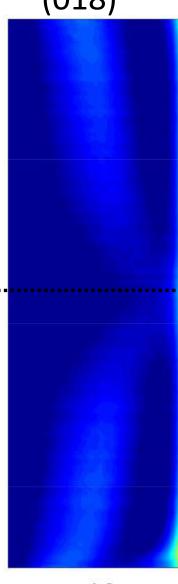

$18 \quad 18.2$
Al (110)

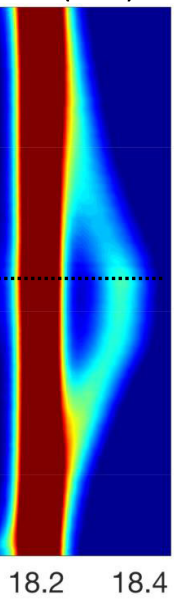

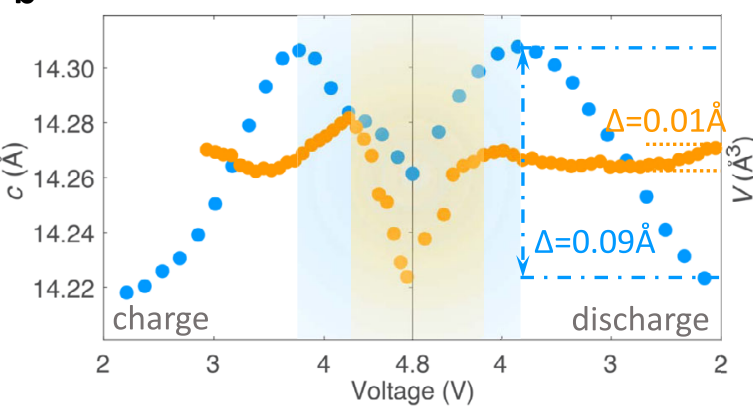

$2 \theta\left({ }^{\circ}\right)$

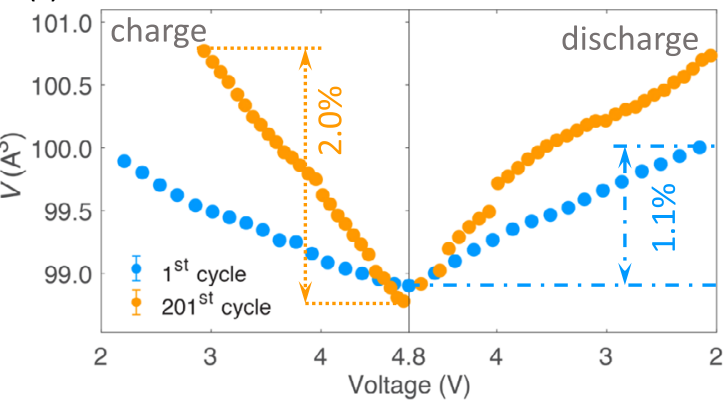

Fig. 6 Comparison of phase evolutions. a Contour plot of in-operando XRD patterns during the $200^{\text {th }}$ cycle. Left figure shows the corresponding chargedischarge curves. Red to blue represents the decreasing peak intensity. $\mathbf{b}$ Comparison of the lattice parameters between the $200^{\text {th }}$ cycle and the first cycle after the electrode activation at a low rate of $0.1 \mathrm{C}$. The lattice parameters ( $c$ and $\mathrm{V}$ ) with errors were obtained by the corresponding in-operando XRD refinement.

from two-phase model fitting complex (Supplementary Fig. 15). The average lattice parameters were obtained by this method, which would not affect the conclusion of sluggish reaction kinetics in aged electrode. These in-operando XANES and XRD results suggest the electrochemical degradation of Li-rich layered oxides originate from the asynchronous reactivity between various TMs and chemo-mechanical instability.

Reaction kinetics. The electronic and physical structural changes investigated above can unequivocally induce sluggish reaction kinetics and lead to electrochemical degradation. Galvanostatic intermittent titration techniques (GITT) and electrochemical impedance spectroscopy (EIS) were used in the first cycle and after long-term cycling (Fig. 7 and Supplementary Figs. 16, 17) to quantify kinetic evolution. The parameters of the charge-transfer resistance, $\mathrm{Li}^{+}$diffusion coefficient $\left(D_{\mathrm{Li}^{+}}\right)$, and reaction overpotential $(\eta)$ were quantified as a function of capacity to investigate electrochemical kinetic variations. The significantly increased semicircle in the high frequency region of EIS curves after cycling (Supplementary Fig. 16) indicates increased electrochemical reaction resistance in the LR-NCM electrode. As a function of discharge capacity, the $D_{\mathrm{Li}^{+}}$(Fig. 7c) and $\eta$ (Fig. $7 \mathrm{~d}$ ) show distinct behaviors at different cycles over the whole $\mathrm{Li}^{+}$ extraction process. Upon the $200^{\text {th }}$ discharge, $D_{\mathrm{Li}^{+}}$values drop sharply, and the overpotential $\eta$ increases up to $100 \mathrm{mV}$. This growing polarization and lithium diffusion decrease are highly correlated to the chemical and electronic structural changes that correspond with the evolution of reaction inhomogeneity as shown above. In this study, we, therefore, reveal the origin of electrochemical degradation and relate structural changes to it with respect to reaction kinetics.

\section{Discussion}

In summary, the spatial structure, which includes spatial electronic and chemo-mechanical structure and spatial electrochemistry, at multiple length scales ranging from atomic to $3 \mathrm{D}$ bulk averages play a major role in battery electrochemical degradation $^{22}$. Current understanding of spatial reactivity in LRNCM material reveals the deactivation of manganese and rearrangement of nickel that results in anisotropic particle reactivity (Fig. 8). This inhomogeneous reactivity leads to small localized lithium diffusion coefficients and augments reaction impedance, which is closely-correlated to electrochemical performance ${ }^{30}$. In turn, sluggish local reaction kinetics could further aggravate local charge inhomogeneity, resulting in increased strain inside the material and eventual mechanical deterioration of the electrode. Non-uniform lithiation states in the material arise from anisotropic reactivity in various transition metals, which causes structural and electrochemical degradation. Anisotropic reactivity and metal rearrangement were therefore revealed as the major reasons for electrochemical degradation in LR-NCM material.

Based on our findings, surface modification combined with doping could greatly resolve electrochemical degradation in LMR. Though, surface modification or doping alone may not fully suppress both capacity loss and voltage decay issues ${ }^{13}$. For example, electrochemical degradation was still observed in LMR materials coated with only $\mathrm{MnO}_{2}{ }^{31}$ or $\mathrm{LiFePO}_{4}{ }^{32}$. However, surface modification with partial doping near the surface area leads to greatly enhanced voltage performance and cycle stability 

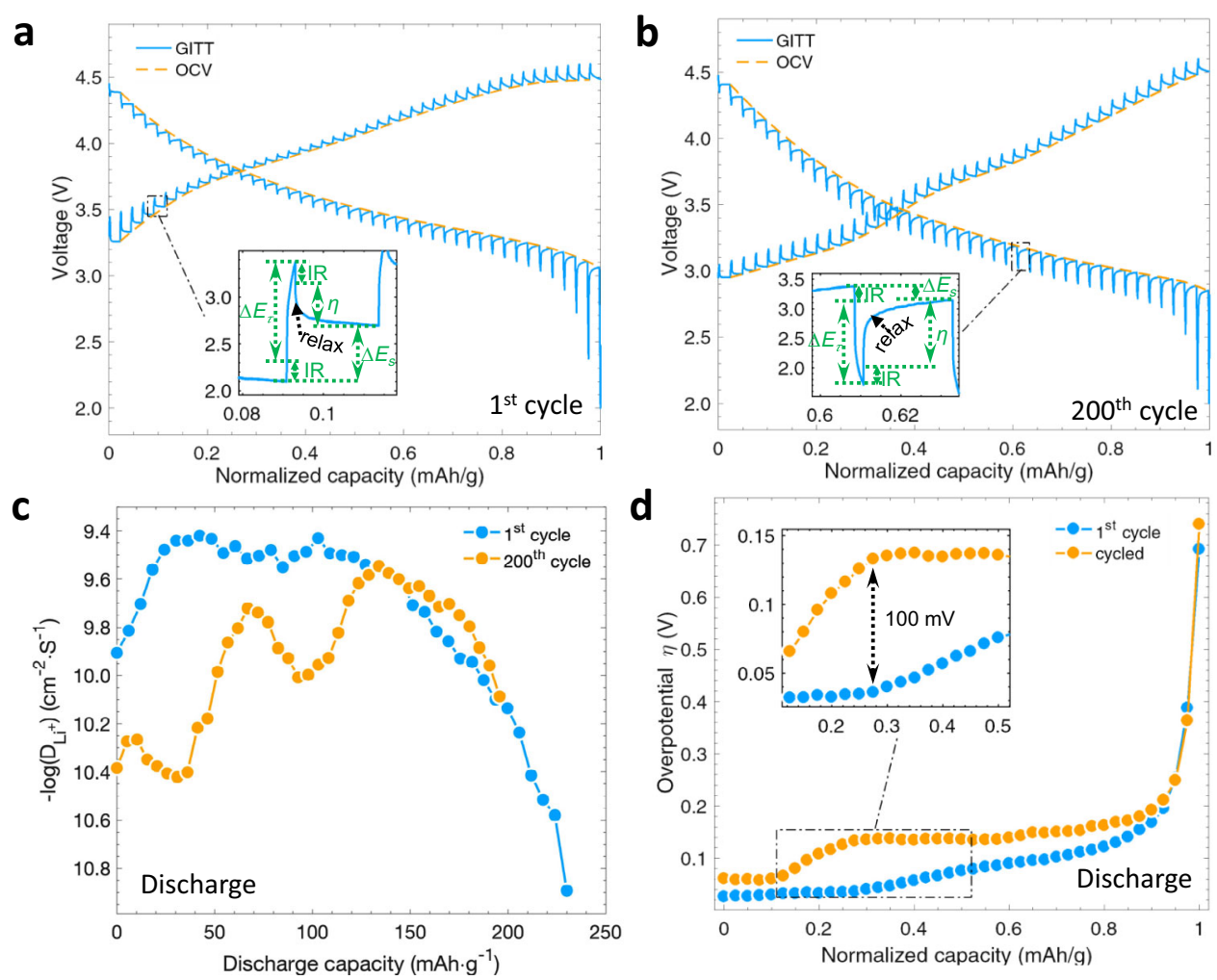

Fig. 7 Evolutions of reaction kinetics. GITT measurements at $\mathbf{a}$ the first cycle after low-rate activation and $\mathbf{b}$ after 200 electrochemical cycles. The open circuit voltage (OCV) obtained after 120 min relaxation at each current pulse was connected with yellow dashed lines. Inserts were the close-up view of the corresponding one current pulse process. IR drops, changes in steady-state voltage $\left(\Delta E_{s}\right)$ and the total battery voltage $\left(\Delta E_{\tau}\right)$ change at one single-step constant pulse were marked to show the main parts that contributed to $\mathrm{Li}^{+}$diffusion coefficient. c The corresponding $\mathrm{D}\left(\mathrm{Li}^{+}\right)$and $\mathbf{d}$ reaction overpotential $(\eta)$ of the electrode at different states during the first and $200^{\text {th }}$ discharge process.
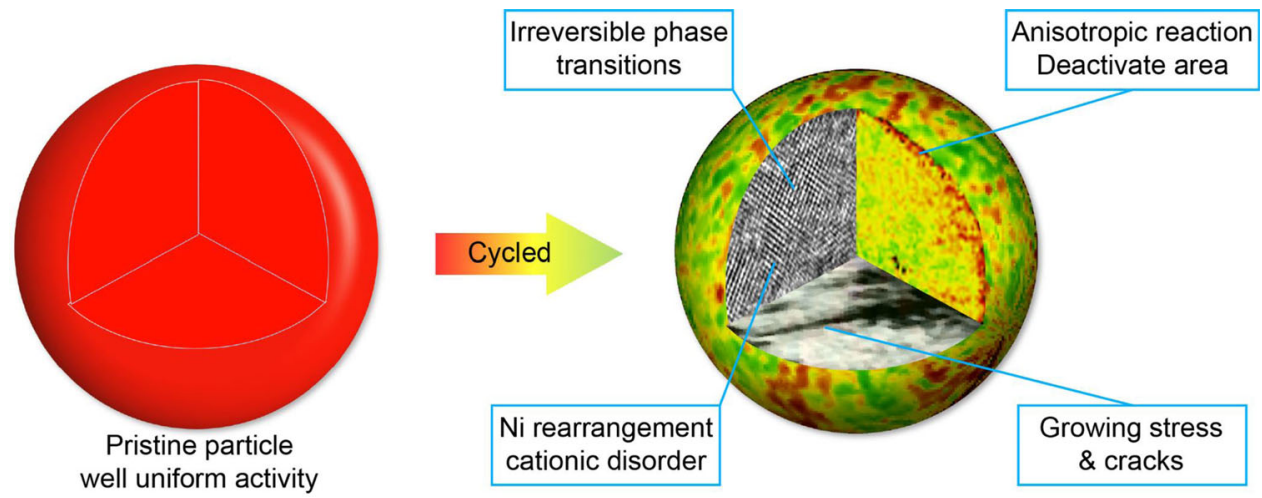

Fig. 8 Schematic illustration of the degradation mechanism.

due to the formation of a stable, uniform, electrochemically active surface $^{16,33}$. A new dual particle doping with surface architecture approach is on the way in our group to address these electrochemical degradation problems in LMR material. Nevertheless, this study offers valuable insights into battery material degradation mechanisms at multiple length-scales for LMR material, which is critical for fundamental research and realization of practical applications.

\section{Methods}

Material preparation. The Li- and Mn-rich cathode material was prepared through a solid phase reaction between precursor and $\mathrm{LiOH}$ at $900^{\circ} \mathrm{C}$ for $20 \mathrm{~h}$ in air atmosphere. $\mathrm{Mn}_{0.666} \mathrm{Ni}_{0.167} \mathrm{Co}_{0.167} \mathrm{CO}_{3}$ precursor was prepared with a traditional co-precipitation method, where $\mathrm{MnSO}_{4} \cdot \mathrm{H}_{2} \mathrm{O}, \mathrm{NiSO}_{4} \cdot \mathrm{H}_{2} \mathrm{O}$, and $\mathrm{CoSO}_{4} \cdot \mathrm{H}_{2} \mathrm{O}$ material, at a molar ratio of 4:1:1, were dissolved in deionized water to obtain a
2.0 M TM solution. This solution was then slowly pumped into a $2 \mathrm{~L}$ tank and continuously stirred under nitrogen atmosphere. The solution $\mathrm{pH}$ value was maintained at 7.5 by the $\mathrm{Na}_{2} \mathrm{CO}_{3}$ and $\mathrm{NH}_{3} \cdot \mathrm{H}_{2} \mathrm{O}$ solutions, and reacted for $12 \mathrm{~h}$ to obtain $\mathrm{Mn}_{0.666} \mathrm{Ni}_{0.167} \mathrm{Co}_{0.167} \mathrm{CO}_{3}$ precursor.

Electrochemical measurements. The electrode was prepared with $80 \mathrm{wt} \%$ active material, $10 \%$ polyvinylidene fluoride, and $10 \%$ carbon black in N-methyl-2pyrrolidinone and coated onto aluminum current collectors. 2032-type coin cells were assembled in an argon-filled glovebox $\left(\mathrm{O}_{2}\right.$ and $\mathrm{H}_{2} \mathrm{O}$ level last than $\left.0.5 \mathrm{ppm}\right)$ using $1 \mathrm{M} \mathrm{LiPF} 6$ in a mixture of ethylene carbonate and dimethyl carbonate (1:1 by volume) as the electrolyte. All the batteries used in this work were tested by the MACCOR battery cycler at room temperature. The cycle performance of LR-NCM was measured at acurrent of $0.3 \mathrm{C}$ for 200 cycles right after three activation cycles at a low rate of $0.1 \mathrm{C}$.

Physical characterization. In-situ X-ray absorption spectroscopy experiments were conducted at beamline 9-BM-C and 12-BM-B, advanced photon source 
(APS), Argonne National Laboratory (ANL), at Ni and Mn K-edge through the transmission mode. XAS data were normalized, Fourier transformed, and analyzed using Athena and Artemis packages. In-situ X-ray diffraction experiments were performed on the LR-NCM electrode during the $200^{\text {th }}$ cycle at beamline $17-\mathrm{BM}$ with the $\mathrm{X}$-ray wavelength of $0.4526 \AA$. The XRD patterns were integrated and analyzed using GSAS-II packages. A coin cell with $3 \mathrm{~mm}$ Kapton window was used for in-situ XANES and in-situ XRD measurements (see the cell design in Supplementary Fig. 18). The insitu experiments should be carefully design and setup to avoid the detrimental effects caused by the non-conductivity Kapton window on the in-situ cell. In this study, we used the normal electrode design with aluminum as the current collector to achieve high electric conductivity and to eliminate the detrimental effects. Advanced in-situ battery cell AMPIX that developed by APS, ANL, would also much help to remove these effects ${ }^{34}$. Full-field transmission X-ray microscopy data were collected at beamline 32-ID-C and the experimental details for $2 \mathrm{D}$ chemical mappings and $3 \mathrm{D}$ nano-tomography are described as in the following section.

\section{Full-field transmission X-ray microscopy}

2D TXM measurement and data analysis. The ex-situ 2D TXM-XANES chemical mappings were collected at both $\mathrm{Ni}(8333 \mathrm{eV})$ and $\mathrm{Mn}(6539 \mathrm{eV}) \mathrm{K}$-edge ${ }^{35}$. To record the phase transformations in the material, a full series of XANES images were collected on the material at pristine and after cycled. The full XANES images were collected by scanning $K$-edge (e.g., Mn, $6539 \mathrm{eV}$ ) from 6519 to $6639 \mathrm{eV}$ with an energy step size of $1 \mathrm{eV}$, which generated $1024 \times 1024$ XANES spectra, corresponding to $\sim 30 \mathrm{~nm}$ output pixel size. The exposure time for each XANES image was 2 seconds. The background images collected at each energy were applied on all the corresponding XANES images. Then, we can extract the full XANES spectrum (X-ray intensity vs energy) for each pixel. Based on Beer's Law, the attenuation coefficient $\mu$ for the given phase and thickness $t$ could be defined as:

$$
\frac{I}{I_{0}}=\exp (-\mu(\mathrm{E}) t)=\exp \left(-\mu_{\text {pristine }} t_{\text {pristine }}\right) \cdot \exp \left(-\mu_{\text {cycled }} t_{\text {cycled }}\right)
$$

where $I_{0}$ is the incident X-ray intensity and $I$ is corresponding X-ray intensity after the attenuating phase. Note that $\mu$ is a function of energy and can be attributed to the two pristine and cycled states.

The scaled $-\ln \left(I / I_{0}\right)$ at each pixel was then fitted by the linear combination of two $\mu$ values. The ratio of the weighting factor is analogous to the thickness fraction and therefore represents their volume fraction.

$$
-\ln \left(\frac{I}{I_{0}}\right)=\mu_{\text {pristine }} t_{\text {pristine }}+\mu_{\text {cycled }} t_{\text {cycled }}
$$

The spectrum fitting was carried out by minimizing the measure of misfit ( $R$ value) for each spectrum at each pixel, which is defined as:

$$
\mathrm{R}=\sum_{\mathrm{Ei}}^{\mathrm{Ef}}(\mathrm{dataE}-\mathrm{refE})^{2} / \sum_{\mathrm{Ei}}^{\mathrm{Ef}} \mathrm{dataE}^{2}
$$

where Ei and Ef are the beginning and ending scan energy (Ni: 8313-8413 eV, Mn: $6519-6619 \mathrm{eV}$ ), respectively. dataE is the normalized spectrum for the given energy $\mathrm{E}$ at each pixel, and refE is the possible fitting reference value that is a linear combination of X-ray attenuation of the pristine and cycled material. Detailed experimental setup and principles and data processing of 2D TXM-XANES mappings can be found in Supplementary Fig. 2 .

$3 D$ nano-tomography. 3D elemental distribution on the secondary particles was obtained by calculating the difference between below and above X-ray absorption edge. All the $3 \mathrm{D}$ nano-tomography data were collected at the energies of below and above absorption edge (Mn: $6530 \mathrm{eV}$ and $6565 \mathrm{eV}, \mathrm{Ni}: 8313 \mathrm{eV}$ and $8360 \mathrm{eV})$ to resolve the $\mathrm{Mn}$ and Ni distribution. The $3 \mathrm{D}$ distribution of TMs can be achieved by subtracting the data of low energy from the data of above absorption edge. 1021 projection images were collected for each sample over an angular range of $180^{\circ}$ from $-90^{\circ}$ to $90^{\circ}$ enabling high spatial resolution of sub- $40 \mathrm{~nm}$. The $3 \mathrm{D}$ nanotomography data were reconstructed from these two-dimensional projections with in house code running on Python.

\section{Data availability}

All the relevant data are available within the paper and its Supplementary Information file or from the corresponding author upon reasonable request.

Received: 21 January 2021; Accepted: 18 August 2021; Published online: 10 September 2021

\section{References}

1. Assat, G. \& Tarascon, J.-M. Fundamental understanding and practical challenges of anionic redox activity in Li-ion batteries. Nat. Energy 3, 373-386 (2018).
2. Erickson, E. M. et al. Review-recent advances and remaining challenges for lithium ion battery cathodes II. Lithium-rich, $\mathrm{xLi}_{2} \mathrm{MnO}_{3} \cdot(1-\mathrm{x})$ $\mathrm{LiNi}_{\mathrm{a}} \mathrm{Co}_{\mathrm{b}} \mathrm{Mn}_{\mathrm{c}} \mathrm{O}_{2}$. J. Electrochem Soc. 164, A6341-A6348 (2017).

3. Manthiram, A. A reflection on lithium-ion battery cathode chemistry. Nat. Commun. 11, 1550 (2020)

4. Li, M. \& Lu, J. Cobalt in lithium-ion batteries. Science 367, 979-980 (2020).

5. $\mathrm{Xu}, \mathrm{X}$. et al. Radially oriented single-crystal primary nanosheets enable ultrahigh rate and cycling properties of $\mathrm{LiNi}_{0.8} \mathrm{Co}_{0.1} \mathrm{Mn}_{0.1} \mathrm{O}_{2}$ cathode material for lithium-ion batteries. Adv. Energy Mater. 9, 1803963 (2019).

6. Rozier, P. \& Tarascon, J. M. Review-Li-rich layered oxide cathodes for nextgeneration Li-ion batteries: chances and challenges. J. Electrochem Soc. 14, A2490-A2499 (2015)

7. Nakayama, K., Ishikawa, R., Kobayashi, S., Shibata, N. \& Ikuhara, Y. Dislocation and oxygen-release driven delithiation in $\mathrm{Li}_{2} \mathrm{MnO}_{3}$. Nat. Commun. 11, 4452 (2020).

8. Yin, W. et al. Structural evolution at the oxidative and reductive limits in the first electrochemical cycle of $\mathrm{Li}_{1.2} \mathrm{Ni}_{0.13} \mathrm{Mn}_{0.54} \mathrm{Co}_{0.13} \mathrm{O}_{2}$. Nat. Commun. 11, 1252 (2020).

9. Li, M. et al. Cationic and anionic redox in lithium-ion based batteries. Chem. Soc. Rev. 49, 1688-1705 (2020).

10. $\mathrm{Hu}, \mathrm{E}$. et al. Evolution of redox couples in $\mathrm{Li}$ - and $\mathrm{Mn}$ - rich cathode materials and mitigation of voltage fade by reducing oxygen release. Nat. Energy 3, 690-698 (2018).

11. Ning, F. et al. Inhibition of oxygen dimerization by local symmetry tuning in Li-rich layered oxides for improved stability. Nat. Commun. 11, 4973 (2020).

12. Li N. et al. Enabling facile anionic kinetics through cationic redox mediator in Li-rich layered cathodes. ACS Energy Lett. 5, 3535-3543 (2020).

13. Cui, C. et al. Structure and interface design enable stable Li-rich cathode. $J$. Am. Chem. Soc. 142, 8918-8927 (2020).

14. Gent, W. E. et al. Coupling between oxygen redox and cation migration explains unusual electrochemistry in lithium-rich layered oxides. Nat. Commun. 8, 2091 (2017).

15. Luo, K. et al. Charge-compensation in 3d-transition-metal-oxide intercalation cathodes through the generation of localized electron holes on oxygen. Nat. Chem. 8, 684-691 (2016).

16. Zhu, Z. et al. Gradient Li-rich oxide cathode particles immunized against oxygen release by a molten salt treatment. Nat. Energy 4, 1049-1058 (2019)

17. Wang, L. et al. Structural distortion induced by manganese activation in a lithium-rich layered cathode. J. Am. Chem. Soc. 142, 14966-14973 (2020).

18. Yuan, Y. \& Lu, J. Demanding energy from carbon. Carbon Energy 1, 8-12 (2019).

19. Singer, A. et al. Nucleation of dislocations and their dynamics in layered oxide cathode materials during battery charging. Nat. Energy 3, 641-647 (2018).

20. Assat, G., Iadecola, A., Foix, D., Dedryvère, R. \& Tarascon, J.-M. Direct quantification of anionic redox over long cycling of Li-rich NMC via hard X-ray photoemission spectroscopy. ACS Energy Lett. 3, 2721-2728 (2018).

21. Yang Z. et al. Probing dopant redistribution, phase propagation, and local chemical changes in the synthesis of layered oxide battery cathodes. Adv. Energy Mater. 11, 2002719 (2020)

22. Wang, L., Wang, J. \& Zuo, P. Probing battery electrochemistry with in operando synchrotron X-ray imaging techniques. Small Methods 2, 1700293 (2018).

23. Wang, L. et al. Unravelling the origin of irreversible capacity loss in $\mathrm{NaNiO}_{2}$ for high voltage sodium ion batteries. Nano Energy 34, 215-223 (2017).

24. Zhan, C., Wu, T., Lu, J. \& Amine, K. Dissolution, migration, and deposition of transition metal ions in Li-ion batteries exemplified by Mn-based cathodes - a critical review. Energy Environ. Sci. 11, 243-257 (2018).

25. House R. A. et al. The role of $\mathrm{O}_{2}$ in O-redox cathodes for Li-ion batteries. Nat. Energy 6, 781-789 (2021).

26. Phillips, P. J., Bareño, J., Li, Y., Abraham, D. P. \& Klie, R. F. On the localized nature of the structural transformations of $\mathrm{Li}_{2} \mathrm{MnO}_{3}$ following electrochemical cycling. Adv. Energy Mater. 5, 1501252 (2015).

27. Hua, W. et al. Structural insights into the formation and voltage degradation of lithium- and manganese-rich layered oxides. Nat. Commun. 10, 5365 (2019).

28. Yabuuchi, N., Yoshii, K., Myung, S.-T., Nakai, I. \& Komaba, S. Detailed studies of a high-capacity electrode material for rechargeable batteries, $\mathrm{Li}_{2} \mathrm{MnO}_{3}-\mathrm{LiCo}_{1 / 3} \mathrm{Ni}_{1 / 3} \mathrm{Mn}_{1 / 3} \mathrm{O}_{2}$. J. Am. Chem. Soc. 133, 4404-4419 (2011).

29. Croy, J. R. et al. First-charge instabilities of layered-layered lithium-ionbattery materials. Phys. Chem. Chem. Phys. 17, 24382-24391 (2015).

30. Chen, H. \& Islam, M. S. Lithium extraction mechanism in Li-rich $\mathrm{Li}_{2} \mathrm{MnO}_{3}$ involving oxygen hole formation and dimerization. Chem. Mater. 28, 6656-6663 (2016)

31. Guo S. et al. Surface coating of lithium-manganese-rich layered oxides with delaminated $\mathrm{MnO}_{2}$ nanosheets as cathode materials for Li-ion batteries. J. Mater. Chem. A 2, 4422-4428 (2014). 
32. Zheng, F. et al. Nanoscale surface modification of lithium-rich layered-oxide composite cathodes for suppressing voltage fade. Angew. Chem. 54, 13058-13062 (2015).

33. Zhu Z. et al. Stabilized Co-free Li-rich oxide cathode particles with an artificial surface prereconstruction. Adv. Energy Mater 10, 2001120 (2020).

34. Borkiewicz, O. J. et al. The AMPIX electrochemical cell: a versatile apparatus for in situ X-ray scattering and spectroscopic measurements. J. Appl Cryst. 45, 1261-1269 (2012).

35. Wang, L. et al. Understanding the initial irreversibility of metal sulfides for sodium-ion batteries via operando techniques. Nano Energy 43, 184-191 (2018).

\section{Acknowledgements}

The work at Argonne National Laboratory was supported from the Clean Vehicles, USChina Clean Energy Research Centre (CERC-CVC2) under the U.S. Department of Energy (DOE), Office of Energy Efficiency and Renewable Energy, Vehicle Technologies Office. Argonne National Laboratory is operated for DOE Office of Science by UChicago Argonne, LLC, under contract number DE-AC02-06CH11357. This research used 9-BM, 11-ID-C, 12-BM, 17-BM, and 32-ID-C beamline at Advanced Photon Source, a U.S. Department of Energy (DOE) Office of Science User Facility operated for the DOE Office of Science by Argonne National Laboratory under Contract No. DE-AC02-06CH11357. This work was supported by the National Natural Science Foundation of China (51872209 and 52072273).

\section{Author contributions}

L.W. and J.L. conceived the ideas and designed the experiments. L.W., T.L.T.W., V.D.A., Y.R., W.X., and S.L. carried out the synchrotron-based experiments and analyzed the data; L.W., A.D., and H.L. prepared the materials and conducted the electrochemical measurements; Q.Z. and L.G. conducted the TEM experiments, H.J., and S.W. discussed the results. L.W., T.L., A.L. S.W., and J.L. wrote the manuscript. J.L. supervised the project.

\section{Competing interests}

The authors declare no competing interests.

\section{Additional information}

Supplementary information The online version contains supplementary material available at https://doi.org/10.1038/s41467-021-25686-1.

Correspondence and requests for materials should be addressed to Tianpin $\mathrm{Wu}$, Huile Jin or Jun Lu.

Peer review information Nature Communications thanks Karin Kleiner and the other, anonymous, reviewer(s) for their contribution to the peer review of this work.

Reprints and permission information is available at http://www.nature.com/reprints

Publisher's note Springer Nature remains neutral with regard to jurisdictional claims in published maps and institutional affiliations.

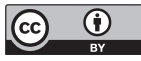

Open Access This article is licensed under a Creative Commons Attribution 4.0 International License, which permits use, sharing, adaptation, distribution and reproduction in any medium or format, as long as you give appropriate credit to the original author(s) and the source, provide a link to the Creative Commons license, and indicate if changes were made. The images or other third party material in this article are included in the article's Creative Commons license, unless indicated otherwise in a credit line to the material. If material is not included in the article's Creative Commons license and your intended use is not permitted by statutory regulation or exceeds the permitted use, you will need to obtain permission directly from the copyright holder. To view a copy of this license, visit http://creativecommons.org/ licenses/by/4.0/.

This is a U.S. Government work and not under copyright protection in the US; foreign copyright protection may apply 2021 